\title{
La educación a distancia desde el pensamiento sistémico: una mirada necesaria para los actores del centro educativo de nivel superior
}

Mauro Marino-Jiménez, Ursula-Andrea Harman-Canalle y Francisco Alvarado-Choy

\section{RESUMEN}

La evolución de la educación a distancia (ED) ha satisfecho diversas necesidades educativas, gracias al progreso tecnológico, iniciativas docentes en la educación superior e investigación educativa. Sin embargo, en la ED confluyen múltiples relaciones entre las capacidades, formas de aprendizaje, participación en la toma de decisiones de los actores y agentes involucrados, normas y valores, entre otros. En tal sentido, el presente artículo tiene como objetivo evidenciar la complejidad de las relaciones entre los elementos del sistema de ED a través del pensamiento sistémico. Esto permitirá explicar por qué la gestión de la ED en centros educativos de educación superior no siempre ha tenido los resultados esperados, y reflexionar sobre la toma de decisiones bajo este marco de referencia.

Palabras clave: administración educativa, enseñanza a distancia, convergencia de sistemas educativos, tecnologías para el aprendizaje y el conocimiento (TAC), tecnologías de la información y de la comunicación (TIC).

\section{Mauro Marino-Jiménez}




\section{A educação a distância segundo o pensamento sistêmico: um olhar necessário para os atores do centro educativo de nível superior}

\section{RESUMO}

A evolução da educação a distância (ED) tem satisfeito diversas necessidades educativas, graças ao progresso tecnológico, iniciativas docentes na educação superior e pesquisa educativa. Porém, na ED confluem múltiplas relações entre as capacidades, formas de aprendizagem, participação na toma de decisões dos atores e agentes envolvidos, normas e valores, entre outros. Nesse sentido, este artigo tem como objetivo evidenciar a complexidade das relações entre os elementos do sistema de ED através do pensamento sistêmico. Isto permitirá explicar por que a gestão da ED em centros educativos de educação superior nem sempre tem tido os resultados esperados; e refletir sobre a toma de decisões sob este marco de referência.

Palavras chave: administração educativa, ensino a distância, convergência de sistemas educativos, tecnologias para a aprendizagem e o conhecimento (TAC), tecnologias da informação e da comunicação (TIC).

\section{Distance education from the systemic way of thinking: a necessary perspective for the actors of higher education institutions}

\section{ABSTRACT}

The evolution of distance education (DE) has met various educational needs, thanks to technological progress, teaching initiatives in higher education and educational research. However, many relationships converge between the capacities, the ways of learning, the participation in the decision making by the actors and the agents involved, the norms and values, among others. This article aims to highlight the complexity of the relationships between the elements of the DE system by means of the systemic way of thinking. This will make it possible to explain why the management of DE in higher education institutions has not always had the expected results and to reflect on decision-making under this framework of reference.

Key words: educational management, distance learning, convergence of educational systems, learning and knowledge technologies (LKT), information and communication technologies (ICT). 


\section{Introducción}

El surgimiento de las tecnologías de la información y la comunicación (TIC) ha potenciado cambios en las prácticas sociales, laborales, investigativas y educativas. Transformaciones que comprometen a la mitad de la población mundial (Internet Live Stats, 2019) y que derivan en un mayor acceso a la educación y oportunidades de desarrollo (ONU, 2018; UNESCO, 2014). Adicionalmente, se puede afirmar que el uso de las TIC también ha dispuesto formas o estilos de trabajo que privilegian, entre otros factores, una mayor velocidad de acceso y producción de información, brevedad de contenidos, preeminencia de lo visual frente a lo escrito, potenciación de la autonomía respecto de la información y desarrollo de comunidades globales (Atif y Chou, 2018; Kim y Choi, 2018; Lan, 2018; Prensky, 2018).

Esta misma tendencia se observa en la literatura del campo de estudio de la ED. En dichas fuentes, se ha encontrado un número creciente de estudios vinculados a prácticas que tienen como protagonista la aplicación de las TIC, tales como el uso de aplicaciones móviles para potenciar resultados de aprendizaje (mobile learning); el empleo de blogs para la producción de contenidos (blogging); el diseño e implementación de cursos masivos en línea (MOOC); el uso de herramientas de gestión de información (Big Data); la experimentación de prácticas a través de un Learning Management System (LMS); la incorporación de la realidad virtual y la percepción estudiantil frente a alguna de estas opciones (Carrera et al., 2018; Kelly, 2018). Desde los estudios de la tecnología educativa, el aporte mayoritario se orienta a cómo mejorar las experiencias de aprendizaje. Los estudiantes son los protagonistas en la ED y los docentes son los responsables de garantizar y conducir los procesos de enseñanza y aprendizaje.

Otros autores proponen la participación de distintos aspectos más allá del diseño, uso e impacto de nuevas tecnologías para fines pedagógicos. Éstos se encuentran vinculados con la actuación humana, tales como necesidades educativas en un contexto determinado, los vínculos de los propios estudiantes con una comunidad de aprendizaje, la gestión educativa, entre otros (Kaiser, 2018; Magen-Nagar y Shonfeld, 2018; Yudes-Gómez, Baridon-Chauve y González-Cabrera, 2018; Vázquez-Cano, 2012). En dichos estudios, aparecen reflexiones teóricas para reafirmar la conjugación de lo educativo y lo tecnológico desde un enfoque sistémico; es decir, en una perspectiva que contempla cómo las acciones de cada parte afectan a las otras de diversas formas. No sólo por normativas o líneas de mando, sino también por la coparticipación de los actores en cada una de las etapas de estos procesos y las consecuencias de sus acciones, dentro del propio sistema.

Si bien la investigación en ED viene introduciendo vínculos con dimensiones adicionales a la tecnológica y pedagógica, todavía existe una limitada atención a la complejidad de la misma; sobre todo, para trasladar la reflexión teórica hacia ejemplos o modelos de gestión educativa. La mayoría de publicaciones asociadas a dicho propósito desarrollan miradas parciales del fenómeno, dejando para estudios posteriores su aplicación en la toma de decisiones, el fortalecimiento de las relaciones de confianza, la gestión del conocimiento o la estrategia y modelo de negocio. En tal sentido, al recorrer las últimas investigaciones donde coinciden la ED y la gestión educativa, se encuentran propuestas de trabajo relacionadas con el uso de la tecnología para predecir resultados (Ma, Yang y Zhou, 2018) o describir la falta de conocimiento de la comunidad académica sobre transformación digital (Leontyeva, 2018; Moreira et al., 2018).

Esta situación motiva una propuesta que sea capaz de abordar la complejidad de la ED; pero con la funcionalidad suficiente para una toma de decisiones efectiva. Una alternativa auspiciosa para este propósito es el pensamiento sistémico (Pastor, 2018).

El presente artículo ofrece una reflexión sobre dos necesidades: la ampliación de los estudios que vinculan la $\mathrm{ED}$ con el pensamiento sistémico y la 
presentación de este último como una estrategia para responder satisfactoriamente a situaciones problemáticas en la gestión educativa. Finalmente, las conclusiones invitan a reflexionar sobre la importancia de este enfoque, para la optimización de procesos similares.

\section{Tendencias actuales de la ED y necesidad de un enfoque sistémico}

La ED tiene un objetivo común con la forma presencial: potenciar la calidad del aprendizaje según la propuesta curricular y el nivel académico establecido. Sin embargo, el proceso de comprensión, adaptación y aplicación de sus particularidades (tales como la mediación de las TIC, el establecimiento de normativas específicas, la formación de una cultura digital, la sostenibilidad económica, entre otras), genera la creación de un nuevo sistema, el cual reúne variables adicionales a las de su contraparte más tradicional (Gros et al., 2016; Moore, 2016).

Actuar con estas consideraciones resulta especialmente necesario cuando las prácticas del centro educativo de nivel superior se fundamentan en la modalidad presencial, sus jerarquías conformadas previamente excluyen de participación a los actores involucrados y las comunicaciones entre los departamentos son precarias o unilaterales (Moriello, 2005; Vázquez-Cano, 2012). En tal sentido, al implementar un sistema de ED, resulta igualmente necesario superar formas de trabajo lineal, alimentadas por "soluciones" a corto plazo y el protagonismo de quienes las promueven. Todo eso, desatendiendo los verdaderos objetivos (Arnold y Wade, 2015). Al respecto, Morin declara:

Uno de los principios de la complejidad señala que todo lo que no se regenera se degenera, es preciso regenerarse para no degenerar. Este principio, que se manifiesta claramente en la complejidad biológica, en la regeneración de las células y la autoeco-organización de la vida, es igualmente válido para los conocimientos y los sistemas de ideas. La racionalidad degenera en racionalización cuando abandona el diálogo incierto e inacabable con lo real; la teoría degenera en doctrina cuando expulsa los argumentos que la contradicen o las evidencias que la refutan; el pensamiento complejo degenera en pensamiento simplificante cuando renuncia a la búsqueda permanente de una racionalidad abierta y auto-crítica (Morin, 2017: 163).

La postura de Morin, aplicada a los diversos campos del conocimiento, sugiere que los procesos de cualquier forma de organización deben gestionarse de forma sistémica. Por lo tanto, el diálogo abierto entre agentes (líderes o propulsores de cambios), actores (participantes) y facilitadores (generadores de condiciones) debe asumirse como una parte significativa del proceso. Esto incluye el punto de vista de la participación en conjunto y la instauración de formas que satisfagan una interrelación positiva y consecuente entre las actuaciones y lo que se propone conseguir con éstas (Costamagna y Larrea, 2017). Dicha necesidad se hace más clara en organizaciones humanas, caracterizadas por su complejidad, jerarquía, no linealidad, dinamismo, autoorganización y caos. Es decir, donde los resultados obtenidos corresponden a una cadena de decisiones y eventos difíciles de determinar a corto plazo (Vázquez-Cano, 2012).

Debido al carácter dinámico de la ED, el cual se hace patente en el enriquecimiento del acervo a través de prácticas, investigaciones y nuevas tendencias, resulta pertinente realizar un recorrido que la caracterice y diferencie de otros sistemas. Ello permitirá el análisis y ejemplificación posteriores, mediante procesos del pensamiento sistémico.

En el presente recorrido sobre estudios vinculados con la educación a distancia, se busca compartir la diversidad de investigaciones; es decir, tanto aquellas que se abocan a la mejora de la praxis específica, como a las que buscan la coparticipación en las distintas etapas del desarrollo del modelo educativo. Por tal motivo, los 
estudios se agrupan a través de las tendencias que se han formulado recientemente y que dan pie a una mayor comprensión del fenómeno estudiado.

La primera tendencia, encontrada en la literatura reciente, es el compromiso o apertura que los docentes asumen frente a las TIC; como se evidencia en los trabajos de Arancibia (2018), Bañuls (2018), Joo y Park (2018) y Lores y Sánchez (2018). En todos ellos, se parte de la premisa de que uno de los principales motivos del éxito o fracaso en la ED y la educación asistida con tecnología, es el conocimiento y disposición del docente frente a la tecnología educativa. Por ello, más allá del esfuerzo para conseguir nuevas y mejores herramientas tecnológicas, es indispensable la disposición de quienes protagonizan la concepción, diseño e implementación de un sistema de ED. Un proceso que, en todas sus etapas, incluye la transferencia de conocimiento, la concientización de la propuesta institucional y el diálogo entre todas las instancias implicadas para el propio desarrollo del sistema (Bezuidenhout, 2018).

Una segunda tendencia, generada por la necesidad de ofrecer estándares de calidad educativa, es la triada conformada por la vigencia, la relevancia y la aplicabilidad de la propuesta educativa. Vigencia, porque la inserción de estrategias educativas en la ED implica conocer las características actuales de la población, incluyendo la posición que ésta tiene frente a la tecnología. Relevancia, porque el aprendizaje no se circunscribe a un contexto físico; sino que se desarrolla a través de los propios sistemas informáticos, a través de un entorno cambiante y para el que el estudiante debe estar preparado. Aplicabilidad, porque en todo modelo educativo se deben administrar eficientemente los recursos disponibles, sobre la base del contexto previamente analizado y la capacidad de los protagonistas para afrontar futuras contingencias.

En relación con la vigencia, se considera pertinente mencionar estudios como los de Amorós (2018) y Tydaly y Pérez (2018), en los que se analiza el perfil del estudiante como una forma para construir o mejorar la praxis educativa. Evidentemente, aunque se cuente con un personal debidamente capacitado y comprometido, lo que éste pueda hacer depende también de las características cognitivas, costumbres y motivaciones propias de quienes aprenden.

De forma complementaria a lo anterior, la ED también es considerada en su relevancia. Por ejemplo, Magen-Nagar y Shonfeld (2018) analizaron el grado de apertura de un conjunto de estudiantes frente a un escenario multicultural, a través del uso de herramientas de contenido abierto. Es decir, una vez que contaron con el conocimiento de la población atendida, emplearon una metodología para que el horizonte de aprendizaje se desarrolle con nuevas y más grandes posibilidades. Un caso similar es el propuesto por la investigación sobre ciber-acoso de Yudes-Gómez et al. (2018). En dicho estudio, se analizaron prácticas sociales negativas haciendo uso de la tecnología. Un tema de suma importancia para el aprendizaje y la propia seguridad de los estudiantes.

Asimismo, en cuanto a la aplicabilidad, se analizan situaciones de insuficiencia o mejor aprovechamiento de recursos para gestionar eficientemente un sistema de ED. Un ejemplo de lo primero es el caso de la investigación de Baltodano y Quesada (2018) respecto de las condiciones de acceso a tecnologías por parte de las comunidades indígenas de Costa Rica. En relación con el mejor uso de las TIC, también se menciona el aporte de Meneses et al. (2018), cuya propuesta de un software libre, adaptado a sus necesidades, superó a la eficacia de una plataforma de pago para la enseñanza de las matemáticas.

Una tercera tendencia (que se suma a las características de los docentes y el trabajo sostenido en vigencia, relevancia y aplicabilidad) es la confluencia de estrategias y métodos para mejorar el aprendizaje a través de la tecnología educativa. Este apartado es variado, pues confluyen ejemplos vinculados con estrategias cognitivas, la contribución de otras disciplinas para la optimización de la praxis o el acercamiento a situaciones de contexto real. 
En cuanto a las estrategias cognitivas, se presenta la incorporación y evaluación de una pedagogía digital, desarrollada por Jesson et al. (2018), como alternativa para el desarrollo de prácticas de lectura y escritura en distintos centros educativos. Rufo (2018) hace lo propio en relación al éxito obtenido mediante la combinación del aprendizaje adaptativo (una metodología del siglo XX) y un LMS.

La contribución de otras disciplinas se nutre de experiencias como las de Yang y Quadir (2018), respecto del juego de roles para superar la ansiedad en la enseñanza de lenguas, combinando la psicología y la didáctica en una simbiosis positiva. Un caso similar lo ofrecen Capilla, Gutiérrez y Cubo (2018), cuando analizan las características psicológicas y lingüísticas de prácticas vinculadas al uso de la tecnología. En ambos casos, resulta plausible que el pensamiento y las prácticas culturales ya no pueden ser estudiadas por separado de la tecnología.

Finalmente, en cuanto a la simulación de situaciones de contexto real, se presenta la propuesta de gamificación, presentada por Rusman, Tenier y Specht (2018), para representar situaciones más cercanas a la realidad de los estudiantes, sugiere una mayor afinidad entre éstos y los nuevos saberes. Gelvez y Ossa (2018) desarrollaron juegos de simulación, dedicados a fortalecer las habilidades de estudiantes de pregrado respecto de la administración financiera. Ambos estudios asumieron el aprendizaje como protagonista del hecho educativo.

Estas tres tendencias, que abarcan el compromiso docente, el desarrollo de estándares de calidad (vigencia, relevancia y aplicabilidad) y la confluencia de estrategias y métodos, se vinculan eficientemente con la generación de buenas prácticas educativas. Sin embargo, existen otras por mencionar y promover.

Una cuarta tendencia, consecuente con la apertura que posibilita el uso de la tecnología, es la creciente producción del estudiante; considerando aquí la elaboración de trabajos publicados en línea, y el aprovechamiento del bagaje del mismo para difundir sus propios conocimientos.
Entre los ejemplos de producción estudiantil, se ofrece el estudio de Yeh (2018) para el fortalecimiento del aprendizaje de una segunda lengua y el trabajo intensivo de la autocorrección de contenidos. Por su parte, Chang, Chou y Liang (2018) proponen el desarrollo de portafolios digitales por parte de estudiantes universitarios. En este caso, los beneficios son plausibles para el propio aprendizaje y en el desarrollo de modelos para que las futuras generaciones puedan tomar como referencia.

En cuanto al aprovechamiento del bagaje estudiantil, se puede mencionar el estudio presentado por Osuna-Acedo, Marta-Lazo y Frau Meigs (2018), en el que se desarrolló la transferencia de conocimientos mediante el diseño de cursos por parte de los propios estudiantes. Este último aspecto presenta un importante valor agregado: el entorno virtual, por su propia naturaleza, dispone la exposición de los conocimientos tácitos de los estudiantes, fomentando su empoderamiento y promoviendo la generación de nuevos saberes dentro de una comunidad académica determinada (Siemens, 2017). Por lo tanto, no sólo se genera una descentralización del conocimiento, sino también el fortalecimiento de las conexiones entre todos los actores.

Esta concurrencia de cuatro tendencias, en cuanto a la literatura reciente de la ED, pone de manifiesto una situación de alta complejidad, cuya atención requiere de un enfoque sistémico. Es decir, de una forma de análisis que desarrolle un equilibrio entre la reflexión y la acción. Esta propuesta tiene un antecedente en el trabajo de Moore y Kearsley (2012), quienes identifican inputs y outputs comunes a todo sistema de ED. Entre los primeros, se hallan los vinculados con los actores del hecho educativo (estudiantes y docentes); pero también aquellos vinculados con aspectos administrativos (área administrativa, costes e inversión) y tecnológicos (TIC a utilizar para los cursos, áreas de soporte y evaluación de data obtenida). En cuanto a los segundos, encabezan los resultados evidenciados en los estudiantes (satisfacción, logros 
y retención), junto a los resultados de acreditación y reputación institucional.

Respecto de los inputs (véase cuadro 1), se evidencia un correlato con la literatura revisada. Por ejemplo, conocer las características de los estudiantes, docentes y personal administrativo, corresponde a una mirada necesaria para configurar la propuesta educativa (Amorós, 2018; Baltodano y Quesada, 2018; Bañuls, 2018; Bezuidenhout, 2018; Joo y Park, 2018; Lores y Sánchez, 2018; Magen-Nagar y Shonfeld, 2018).
Precisar la conformación, calidad y características de las asignaturas se condice con el aporte de estrategias educativas y disciplinas vinculadas con el uso de las TIC (Capilla, Gutiérrez y Cubo, 2018; Jesson et al., 2018; Kaiser, 2018; Rufo, 2018; Rusman, Tenier y Specht, 2018; Yang y Quadir, 2018). Finalmente, el aspecto tecnológico, sumado al grado de inversión, no se encuentran entre las investigaciones recientes; sin embargo, resultan inherentes a toda gestión del centro educativo de nivel superior.

Cuadro 1. Inputs de un sistema de ED

\begin{tabular}{|l|l|}
\hline \multicolumn{1}{|c|}{ Dimensiones } & \multicolumn{1}{c|}{ Inputs } \\
\hline \multirow{4}{*}{$\begin{array}{l}\text { Características y } \\
\text { acciones de los actores }\end{array}$} & - Características de los estudiantes, incluyendo su preparación para estudiar a distancia \\
\cline { 2 - 3 } & - Experiencia del docente en educación a distancia \\
\cline { 2 - 3 } Inversión, decisiones y & - Calidad del diseño de las asignaturas \\
\cline { 2 - 3 } control de recursos & - Calidad de la producción de las asignaturas \\
\cline { 2 - 3 } & - Tecnología escogida para las asignaturas \\
\cline { 2 - 3 } & - Costo del diseño y producción de asignaturas \\
\cline { 2 - 3 } & - Frecuencia y calidad de la evaluación de la información \\
\cline { 2 - 3 } &
\end{tabular}

Fuente: adaptado de Moore y Kearsey (2012), traducción de los autores.

Por el lado de los outputs (véase cuadro 2), destaca una triada conformada por satisfacción, puntajes e índice de cumplimiento, lo cual se evidencia en lo que los estudiantes logran desarrollar (Chang, Chou y Liang, 2018; Osuna-Acedo, Marta-Lazo y Frau Meigs, 2018; Yeh, 2018). Inmediatamente después, aparece la evaluación de calidad de la gestión (inscripciones, evaluaciones del trabajo y sostenibilidad). Consecuentemente, la reputación como un indicador que renueva el proceso para el desarrollo de todos los elementos.
La literatura revisada y las listas de inputs y outputs permiten dilucidar las acciones para concebir, diseñar e implementar un sistema de ED en un centro educativo de nivel superior. Sin embargo, la teoría del pensamiento sistémico dispone de más herramientas para el fortalecimiento de dicha gestión. Por tal motivo, el siguiente apartado ofrece un ejemplo de la aplicación de arquetipos en la ED. 
Cuadro 2. Outputs de un sistema de ED

\begin{tabular}{|l|l|}
\hline \multicolumn{1}{|c|}{ Dimensiones } & \multicolumn{1}{c|}{ Outputs } \\
\hline \multirow{4}{*}{ Logros de aprendizaje } & - Satisfacción estudiantil \\
\cline { 2 - 2 } & - Puntajes obtenidos por los estudiantes \\
\cline { 2 - 2 } & - Tasa de finalización del programa académico \\
\hline \multirow{4}{*}{$\begin{array}{l}\text { Gestión y calidad } \\
\text { educativa }\end{array}$} & - Matrículas totales \\
\cline { 2 - 2 } & - Evaluaciones de calidad \\
\cline { 2 - 2 } & - Resultados de la acreditación \\
\cline { 2 - 2 } & - Matrícula y generación de utilidades \\
\hline Reputación & - Reputación y rotación del personal \\
\hline
\end{tabular}

Fuente: adaptado de Moore y Kearsey (2012), traducción de los autores.

\section{Arquetipos en el pensamiento sistémico}

Uno de los principales atributos del pensamiento sistémico es su capacidad para evidenciar las relaciones entre los elementos de un sistema, de modo que se pueda socializar eficazmente dicha información y tomar decisiones con mucha más claridad (Arnold y Wade, 2015; Clancy, 2018; Kim, 2000; Maani y Cavana, 2007, y Senge, 1990). Esta cualidad supone una mirada estratégica a los objetivos de todo el sistema, ya que ilustra cómo relaciones no tomadas en cuenta en un modelo lineal afectan directamente en los resultados.

Este potencial de representación es tributario de una evolución teórica del pensamiento sistémico. Senge (1990), por ejemplo, describe este concepto como una disciplina y un marco de trabajo que permite la comprensión de un sistema en un sentido más profundo. Arnold y Wade (2015) lo consideran como un conjunto de habilidades sinérgicas que permiten desarrollar la capacidad para identificar y entender sistemas; como consecuencia, se tiene la predicción de comportamientos y la realización de modificaciones para conseguir efectos deseables. Finalmente, para Clancy (2018), el término hace referencia a las conexiones entre diferentes elementos y cómo estos producen comportamientos en un sistema complejo y adaptativo.

Bajo esta premisa, la representación de procesos dinámicos a través de arquetipos resulta útil para predecir tendencias en distintos periodos de tiempo. Por ejemplo, una ley para controlar la natalidad de un país tendría consecuencias económicas positivas a corto plazo; sin embargo, al cabo de unas décadas, la población económicamente activa sería proporcionalmente menor y sufriría de medidas severas para sostener la calidad de vida de los jubilados. Un modelo de negocio basado en una tecnología novedosa podría lograr un éxito inicial, basado en la novedad de su servicio; no obstante, un salto cualitativo en otras tecnologías, aprovechadas por la competencia o por los propios usuarios, podrían revertir ese estado inicial. Dichos cambios son plausibles cuando se recurre al pensamiento sistémico. Es decir, cuando se reflexiona sobre el propio sistema, y se desarrolla una visión de la complejidad a la que se enfrentan sus actores (Arnold y Wade, 2015). 
En el caso de la ED, se han identificado las prioridades mediante la relación lineal entre inputs (conocimientos y acciones de la comunidad académica, inversión y control de recursos) y outputs (acciones de los estudiantes, indicadores de gestión y reputación). Sin embargo, los patrones de comportamiento organizacional, ilustrados a través de arquetipos, tienen la virtud de determinar consecuencias (positivas o negativas) y contrarrestar vicios no tan plausibles en el corazón de este sistema.

$\mathrm{El}$ arquetipo Limites de crecimiento (Limits to growth) es elocuente para señalar, por ejemplo, las diferencias entre los resultados deseados y obtenidos. Dicho de otro modo, acciones que suponen un determinado logro van a verse mermadas por condiciones limitantes (internas o externas) que no habían sido consideradas desde un inicio, forzando correcciones y restringiendo las mejoras (véase gráfica 1).

El desconcierto por este resultado puede ser el menor problema que denuncia el arquetipo Limites de crecimiento. En muchas situaciones, cuando la organización opta por redoblar esfuerzos en la dirección equivocada, se puede provocar la extralimitación y el colapso del propio sistema (véase gráfica 2).

\section{Gráfica 1. Arquetipo Límites de crecimiento (Senge, 2006: 137)}

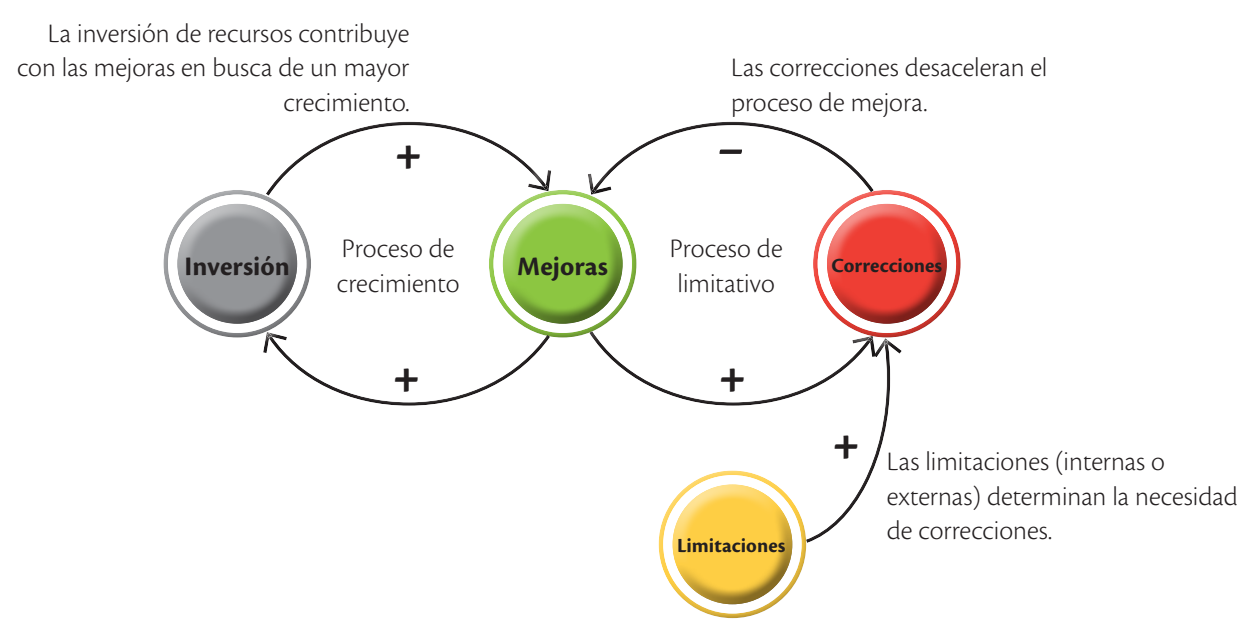

Gráfica 2. Pautas de conducta en el arquetipo Límites de crecimiento (Senge, 2006: 137)

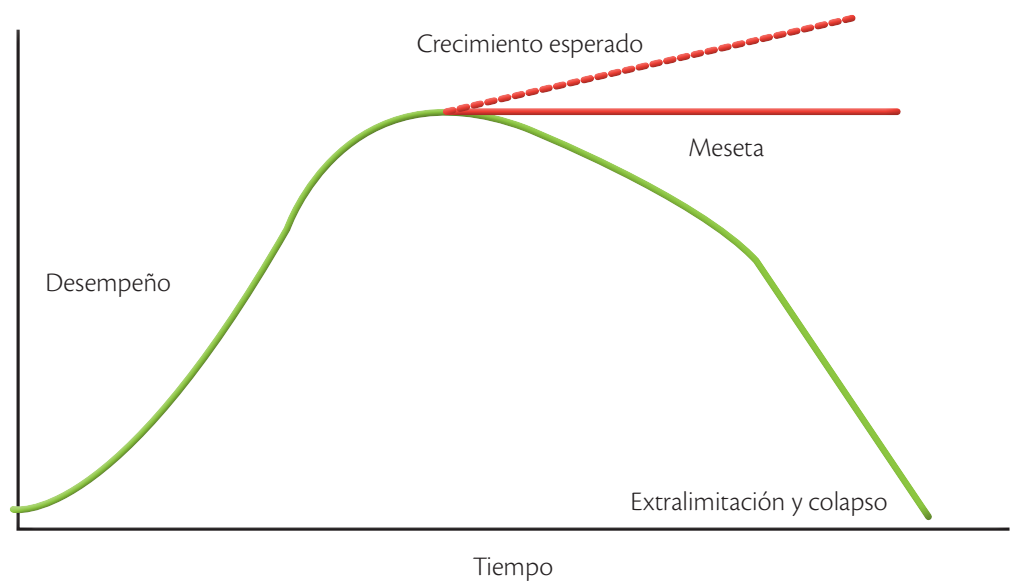


$\mathrm{Al}$ trasladar los Limites del crecimiento al sistema de la ED, se pueden evidenciar, por ejemplo, los problemas generados por prestar atención a la adquisición de tecnología, sin tomar en cuenta un conjunto de limitaciones importantes. Factores como la baja experiencia de los docentes, el tipo de cultura organizacional del centro educativo de nivel superior y la insuficiente normativa sobre la ED, pueden limitar el crecimiento (véase gráfica 3).

\section{Gráfica 3. Arquetipo Límites de crecimiento, aplicado a la ED}

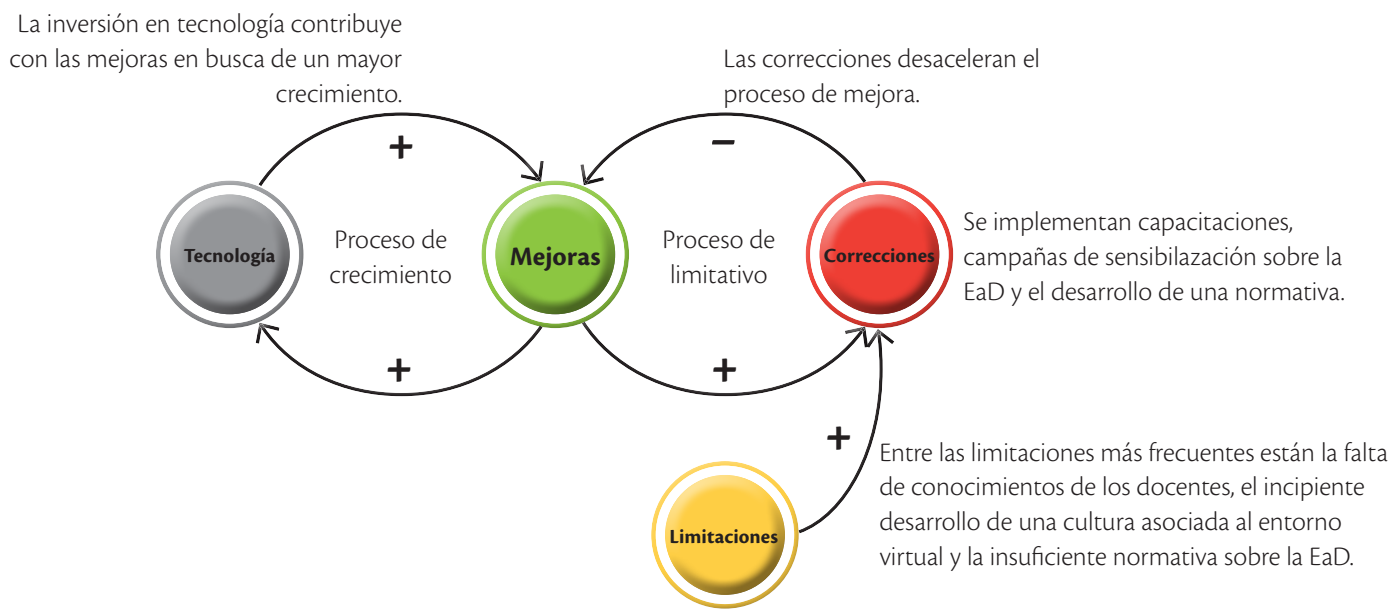

Un sistema complejo debe asumirse de forma igualmente sistémica. Si bien es cierto que incorporar la ED en un centro educativo es una alternativa totalmente válida, esta decisión individual sólo resulta viable a través de un conjunto de actuaciones colectivas, sinérgicamente acompasadas (Behl y Ferreira, 2014; Kim, 2000; Vázquez-Cano, 2012). En tal sentido, si se desestima el alto número de inputs dedicados a lo relacionado con lo que docentes y administrativos conocen, la inversión anticipada en tecnología puede resultar contraproducente.
En cambio, si se prioriza la dedicación al conocimiento de toda la comunidad académica (características de los estudiantes, comprensión del cuerpo administrativo sobre la tecnología educativa y experiencia docente en metodologías digitales), no sólo se trabajará con una inversión menor, sino también con mayor claridad sobre lo que se desea desarrollar en cuanto al diseño de cursos, formas de evaluación y - eventualmente - logro de todos los objetivos (véase gráfica 4 ). 


\section{Gráfica 4. Corrección del arquetipo Límites de crecimiento, aplicado a la ED}

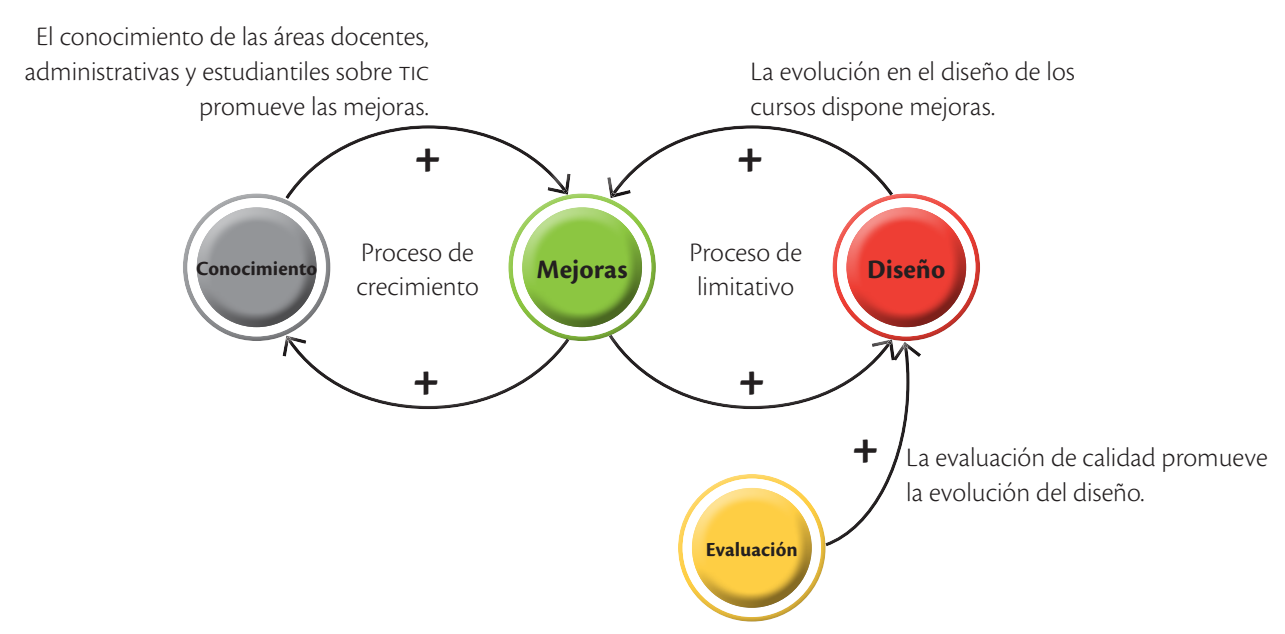

Más aún, para elegir y aprovechar eficientemente la inversión en tecnología, se debe contar con este proceso plenamente establecido. Si bien el uso de equipos y sistemas de última generación puede resultar beneficioso, corresponde mantener la premisa de que la educación es una ciencia social. Outputs como los logros de aprendizaje, la reputación de la institución y las nuevas matrículas se consiguen, principalmente, a través de las interpretaciones de los actores humanos en el centro educativo. Este paso es fundamental para que el sistema se desarrolle con un sano y auténtico crecimiento.

De acuerdo con lo observado, el pensamiento sistémico suma en dos sentidos. Por una parte, brinda los insumos para entender los inputs y outputs de la ED. Por otra, es capaz de brindar las herramientas necesarias para analizar y exponer los procesos dinámicos que existen en la misma. Si bien la visión de linealidad resulta funcional en el ámbito de las máquinas; las organizaciones educativas, que implican aprendizaje e interacción social, se encuentran inmersas en la complejidad de actuaciones humanas que podrían fortalecer o debilitar el sistema. Por lo tanto, las múltiples relaciones entre sus variables resultan cruciales para entender y mejorar su funcionamiento.
Si bien la propuesta ofrecida no se encuentra contextualizada en un estudio de caso, la coincidencia proveniente de la literatura, análisis y procesos resultan adaptables a distintas realidades. A la estructura de los procesos presentados, se deben agregar decisiones específicas ya mencionadas, tales como el conocimiento de la comunidad académica, la elección de la tecnología, la inversión de recursos y la generación de un modelo educativo que declare y defina los objetivos de aprendizaje. Por todo ello, se hace necesario analizar constantemente la visión de conjunto y las implicancias de las interrelaciones entre los elementos del sistema.

\section{Conclusiones}

A partir de la revisión bibliográfica; la identificación de inputs y outputs; y la ejemplificación de un caso de toma de decisiones, haciendo uso de los arquetipos del pensamiento sistémico, este artículo propone la necesidad de analizar la Educación a Distancia como un sistema complejo. Si bien la literatura actual define a la ED como un sistema lineal de inputs y outputs, la originalidad de este artículo reside en entender las complejas interrelaciones entre los elementos de la ED de una manera dinámica. En tal sentido, 
se introduce el pensamiento sistémico como una alternativa para el análisis y reflexión de la ED. Mediante la aplicación del arquetipo Limites del crecimiento, se explican las consecuencias de una toma de decisiones a corto y largo plazo. Por tal motivo, un centro educativo que busca implementar sistemas similares tendrá que buscar el equilibrio entre decidir lo que desea hacer y buscar la participación e interacción de los actores involucrados para saber cómo hacerlo.

La representación de la ED mediante arquetipos, brinda una metodología para estudiar el

\section{Referencias}

Amorós, L. (2018), "Autoevaluación con learning analytics en el Grado Educación Infantil", en F. Carrera, F. Martínez, J. Coiduras, E. Brescó y E. Vaquero (eds.), EDUcación con TECnología: un compromiso social. Aproximaciones desde la investigación y la innovación, Lleida, Edicions de la Universitat de Lleida/Asociación EDUTEG, pp. 142-146, <10.21001/edutec.2018> [Consulta: diciembre de 2018].

Arancibia, M. (2018), "Creencias de los docentes acerca de la enseñanza y su relación con el dominio técnico y didáctico de la plataforma Moodle”, en F. Carrera, F. Martínez, J. Coiduras, E. Brescó y E. Vaquero (eds.), EDUcación con TECnología: un compromiso social. Aproximaciones desde la investigación y la innovación, Lleida, Edicions de la Universitat de Lleida/Asociación EDUTEG, pp. 142-146, <10.21001/edutec.2018> [Consulta: diciembre de 2018].

Arnold, R. D. y J. P. Wade (2015), "A definition of systems thinking: a systems approach", Procedia Computer Science, núm. 44, pp. 669-678.

Atif, Y. y C. Chou (2018), "Guest editorial: digital citizenship: innovations in education, practice, and pedagogy", Educational Technology \& Society, vol. 21, núm. 1, pp. 152-154, <http://www.j-ets.net/ETS/ journals/21_1/14.pdf> [Consulta: diciembre de 2018]. comportamiento de diversos sistemas de gestión de $\mathrm{ED}$, abriendo un nuevo conjunto de estudios afines y contextualizados, en los que se podría representar la toma de decisiones efectuadas, a partir de arquetipos. Finalmente, el pensamiento sistémico permite situar la ED en un contexto de creciente complejidad y dinamismo, en el cual es crucial contar con herramientas que permitan registrar los aprendizajes de la organización y el desarrollo de estrategias para enfrentar los retos del acelerado cambio económico, tecnológico, social y ambiental de la actualidad.
Baltodano, M. y A. Quesada (2018), "Diagnóstico de las condiciones de acceso y uso de tecnologías digitales por parte del profesorado en comunidades indígenas de Costa Rica", en F. Carrera, F. Martínez, J. Coiduras, E. Brescó y E. Vaquero (eds.), EDUcación con TECnología: un compromiso social. Aproximaciones desde la investigación y la innovación, Lleida, Edicions de la Universitat de Lleida/ Asociación EDUTEC, pp. 142-146, <10.21001/ edutec.2018> [Consulta: diciembre de 2018].

Bañuls, G. (2018), "Los docentes, ¿cómo se disponen a aprender sobre el uso de TIC con sentido pedagógico?”, en F. Carrera, F. Martínez, J. Coiduras, E. Brescó y E. Vaquero (eds.), EDUcación con TECnología: un compromiso social. Aproximaciones desde la investigación y la innovación, Lleida, Edicions de la Universitat de Lleida/Asociación EDUTEC, pp 142-146, <10.21001/edutec.2018> [Consulta: diciembre de 2018].

Behl, D. V. y S. Ferreira (2014), "Systems thinking: an analysis of key factors and relationships", Procedia Computer Science, núm. 36, pp. 104-109, <https:// doi.org/10.1016/j.procs.2014.09.045> [Consulta: diciembre de 2018].

Bezuidenhout, A. (2018), "Analysing the importancecompetence gap of distance educators with the increased utilisation of online learning strategies in 
a developing world context", International Review of Research in Open and Distributed Learning, vol. 19, núm. 3, pp. 263-281, <http://www.irrodl.org/index.php/ irrodl/article/view/3585/4706> [Consulta: diciembre de 2018].

Capilla, E., P. Gutiérrez y S. Cubo (2018), "Las redes semánticas naturales como procedimiento para la obtención de la validez de un instrumento que evalúa los comportamientos phubbing", en F. Carrera, F. Martínez, J. Coiduras, E. Brescó y E. Vaquero (eds.), EDUcación con TECnología: un compromiso social. Aproximaciones desde la investigación y la innovación, Lleida, Edicions de la Universitat de Lleida/Asociación EDUTEC, pp.142-146, <10.21001/edutec.2018> [Consulta: diciembre de 2018].

Carrera, F., F. Martínez, J. Coiduras, E. Brescó y E. Vaquero (eds.) (2018), EDUcación con TECnología: un compromiso social. Aproximaciones desde la investigación y la innovación, Lleida, Edicions de la Universitat de Lleida/Asociación EDUTEG, pp. 142-146, <10.21001/edutec.2018> [Consulta: diciembre de 2018].

Chang, C. C., P. N. Chou y C. Liang (2018), "Using ePortfolio-based learning approach to facilitate knowledge sharing and creation among college students", Australasian fournal of Educational Technology, vol. 34, núm. 1, pp. 30-41, <https://doi.org/10.14742/ ajet.2687> [Consulta: diciembre de 2018].

Clancy, T. (2018), "Systems thinking: three system archetypes every manager should know", IEEE Engineering Management Review, vol. 46, núm. 2, pp. 32-41.

Costamagna, P. y M. Larrea (2017), Actores facilitadores del desarrollo territorial. Una aproximación desde la construcción social, Bilbao, Orkestra-Instituto Vasco de Competitividad, <http://www.deusto-publicaciones. es/deusto/pdfs/orkestra/orkestra57.pdf $>$ [Consulta diciembre de: 2018].

Gelvez, L. y L. Ossa (2018), "Evaluación de la viabilidad de los juegos de simulación como recurso de aprendizaje para un programa de pregrado", en F. Carrera et al. (eds.), op. cit. [Consulta: diciembre de 2018].
Gros, B., C. Suárez-Guerrero y T. Anderson (2016), "The internet and online pedagogy editorial”, International Fournal of Educational Technology in Higher Education, núm. 13, <https://doi.org/10.1186/s41239-016-0037-7> [Consulta: diciembre de 2018].

Internet Live Stats (2019), Internet Live Stats, <http:/ /www. internetlivestats.com/> [Consulta: diciembre de 2018]. Jesson, R., S. Mc Naughton, A. Wilson, T. Zhu y V. Cockle (2018), "Improving achievement using digital pedagogy: impact of a research practice partnership", Fournal of Research on Technology in Education, vol. 50, núm. 3, pp. 183-199, <10.1080/15391523.2018.1436012> [Consulta: diciembre de 2018].

Joo, Y. J., S. Park y E. Lim (2018), "Factors influencing preservice teachers' intention to use technology: TPACK, Teacher Self-efficacy, and Technology Acceptance Model", Educational Technology \& Society, vol. 21, núm. 3, pp. 48-59.

Kaiser, A. (2018), "Learning from the future meets Bateson's levels of learning", The Learning Organization, vol. 25, núm. 4, pp. 237-247, <https://doi.org/10.1108/ TLO-06-2017-0065> [Consulta: diciembre de 2018].

Kelly, R. (2018), "7 Ed tech trends to watch in 2018", Campus Technology, <https://campustechnology.com/ Articles/2018/01/11/7-Ed-Tech-Trends-to-Watchin-2018.aspx> [Consulta: diciembre de 2018].

Kim, D. (2000), Systems archetypes, Waltham, Pegasus Communications.

Kim, M. y D. Choi (2018), "Development of youth digital citizenship scale and implication for educational setting", Educational Technology E̊ Society, vol. 21, núm. 1, pp. 155171, <http://www.j-ets.net/ETS/journals/21_1/15. pdf> [Consulta: diciembre de 2018].

Lan, Y. (2018), "Technology enhanced learner ownership and learner autonomy through creation", Education Tech Research Dev, núm. 66, pp. 859-862, <https:// doi.org/10.1007/s1 1423-018-9608-8> [Consulta: diciembre de 2018].

Leontyeva, I. (2018), "Modern distance learning technologies in higher education: Introduction problems", Eurasia Journal of Mathematics, Science and 
Technology Education, vol. 14, núm. 10,pp. 1-8, <10.29333/ ejmste/92284> [Consulta: diciembre de 2018].

Lores, B. y P. Sánchez (2018), "Diseño y validación piloto de un cuestionario sobre el uso, la formación y las actitudes y creencias de docentes de educación primaria de la provincia de Castellón (España)", en F. Carrera et al. (eds.), op. cit. [Consulta: diciembre de 2018].

Ma, X., Y. Yang y Z. Zhou (2018), "Using machine learning algorithm to predict student pass rates in online education", ACM International Conference Proceeding Series, pp. 156-161, <10.1145/3220162.3220188> [Consulta: diciembre de 2018].

Maani, K. y R. Cavana (2007), Systems thinking, system dynamics: managing change and complexity, Auckland, NZ, Prentice Hall.

Magen-Nagar, N. y M. Shonfeld (2018), "Attitudes, openness to multiculturalism, and integration of online collaborative learning", Educational Technology E् Society, vol. 21, núm. 3, pp. 1-11.

Meneses, L., A. García, M. Martínez y M. Peña (2018). "Una experiencia con software libre en la docencia de matemáticas aplicadas a la economía”, en F. Carrera et al. (eds.), op. cit. [Consulta: diciembre de 2018].

Moore, M. G. y G. Kearsley (2012), Distance education: a systems view of online learning, Belmont, CA, Thomson/ Wadsworth.

Moore, M. (2016), "Brazil's PROFORMAÇAO Project: a case study of an integrated virtual system for delivering in-service teacher education", American Fournal of Distance Education, vol. 30, núm. 4, pp. 272274, <10.1080/08923647.2016.1232998> [Consulta: diciembre de 2018].

Moreira, F., N. Durão, C. Pereira y M. J. Ferreira (2018), "Are university professors of the South American countries preparing students for digital transformation?", Advances in Intelligent Systems and Computing, núm. 746, pp. 1296-1306, <10.1007/9783-319-77712-2_124> [Consulta: diciembre de 2018].

Moriello, S. (2005), Inteligencia natural y sintética, Buenos Aires, Editorial Nueva Librería.
Morin, E. (2017), "Reseña de Leonardo G. Rodríguez Zoya (coord..) (2016)", Librarius. Utopía y praxis latinoamericana, vol. 22, núm. 78, pp. 163-174, <http:// www.redalyc.org/articulo.oa?id=27952381013> [Consulta: diciembre de 2018].

Organización de las Naciones Unidas (ONU) (2018), Población, <http://www.un.org/es/sections/issuesdepth/population/index.html> [Consulta: enero de 2019].

Osuna-Acedo, S., C. Marta-Lazo y D. Frau-Meigs (2018), "De sMOOG a tMOOC, el aprendizaje hacia la transferencia profesional: el proyecto europeo ECO”, Comunicar. Revista Científica de Educomunicación, vol. 36, núm. 55, pp. 105-114, <https://doi.org/10.3916/ C55-2018-10> [Consulta: diciembre de 2018].

Pastor, J. (2018), "Liderando el cambio: los cinco estilos de líder transformacional", Harvard Deusto Business Review, núm. 284, pp. 6-21, <https://www.harvarddeusto.com/liderando-el-cambio-los-cinco-estilos-delider-transformacional> [Consulta: diciembre de 2019].

Prensky, M. (2018), "Beyond technology in the classroom: trivial uses vs. powerful uses", en Marc Prensky, $<$ https://marcprensky.com/beyond-technology-inthe-classroom/> [Consulta: diciembre de 2018].

Rufo, J. (2018), "Un caso de éxito de aplicación del aprendizaje adaptativo a una plataforma elearningNeo LMS", en F. Carrera et al. (eds.), op. cit. [Consulta: diciembre de 2018].

Rusman, E., S. Ternier y M. Specht (2018), "Early second language learning and adult involvement in a realworld context: design and evaluation of the 'ELENA goes shopping' mobile game", Educational Technology \& Society, vol. 21, núm. 3, pp. 90-103.

Senge, P. (2006), La quinta disciplina en la práctica, Buenos Aires, Garnica.

Senge, P. (1990), The fifth discipline. The art E practice of the learning organization, Nueva York, Currency Doubleday.

Siemens, G. (2017), "Learning as artifact creation", Elearnspace, <http://www.elearnspace.org/blog/2017 /09/14/learning-as-artifact-creation/> [Consulta: diciembre de 2018]. 
Tydaly, M. y A. Pérez (2018), "Hábitos, usos y actitudes en Internet del alumnado de 8-12 años de la barriada de "Los Junquillos" en la Línea de la Concepción (Cádiz)", en F. Carrera et al. (eds.), op. cit. [Consulta: diciembre de 2018].

UNESCO (2014), Estrategia a plazo medio, París, UNESCO, <http://unesdoc.unesco.org/images/0022/002278/ 227860s.pdf $>$ [Consulta: diciembre de 2018].

Vázquez-Cano, E. (2012), Caos, complejidad y tecnologías en el centro educativo. La escuela en el siglo XXI, Saarbrücken, Editorial Académica Española.

Yang, J. C. y B. Quadir (2018), "Effects of prior knowledge on learning performance and anxiety in an english learning online role-playing game", Educational Technology E̊ Society, vol. 21, núm. 3, pp. 174-185.

Yeh, H.-C. (2018), "Exploring the perceived benefits of the process of multimodal video making in developing multiliteracies", Language Learning \& Technology, vol. 22, núm. 2, pp. 28-37, <https://doi.org/10125/44642> [Consulta: diciembre de 2018].

Yudes-Gómez, C., D. Baridon-Chauve y J. González Cabrera (2018), "Ciberacoso y uso problemático de Internet en Colombia, Uruguay y España: un estudio transcultural", Comunicar. Revista Científica de Educomunicación, vol. 36, núm. 56, pp. 49-58, <https://doi.org/10.3916/ C56-2018-05> [Consulta: diciembre de 2018].

\section{Cómo citar este artículo:}

Marino-Jiménez, Mauro, Ursula-Andrea Harman-Canalle y Francisco Alvarado-Choy (2020), "La educación a distancia desde el pensamiento sistémico: una mirada necesaria para los actores del centro educativo de nivel superior", Revista Iberoamericana de Educación Superior (RIES), vol. XI, núm. 32, pp. 183-197, DoI: https://doi.org/10.22201/iisue.20072872e.2020.32.821 [Consulta: fecha de última consulta]. 